\title{
Metastasis of the epididymis and spermatic cord from pancreatic adenocarcinoma: A rare entity. Description of a case and revision of literature
}

\author{
Carmelo Agostino Di Franco ${ }^{1}$, Bruno Rovereto ${ }^{1}$, Daniele Porru ${ }^{1}$, Valeria Zoccarato ${ }^{1}$, Cesare Regina ${ }^{1}$, \\ Tiziano Cebrelli ${ }^{1}$, Nicolò Fiorello ${ }^{1}$, Alessandra Viglio ${ }^{2}$, Lavinia Galvagno ${ }^{3}$, Carlo Marchetti ${ }^{1}$, \\ Andrea Ringressi $^{1}$, Davide Barletta ${ }^{1}$, Giovanni Giliberto ${ }^{1}$ \\ ${ }^{1}$ Department of Urology, University Hospital IRCCS Policlinico S.Matteo of Pavia, Italy; \\ ${ }^{2}$ Department of Pathology, University Hospital IRCCS Policlinico S.Matteo of Pavia, Italy; \\ ${ }^{3}$ Language and Translation Services, Enna, Italy.
}

\begin{abstract}
Summary Introduction: Metastatic epididymal and spermatic cord adenocarcinoma from epithelial tumors are a rare condition. The most frequent primary cancers are prostate, lung, kidney, gastrointestinal tumors and breast. In literature, there are very low number of cases reporting metastasis from pancreatic cancer to epididymis and spermatic cord.

Case description: We report a case of 70-years old man with history of left orchiectomy for undescended testicle, who presented to our department with a palpable nodule in the right scrotum. Scrotal ultrasound revealed an inhomogeneous hypoechoic nodule of epididymis and/or spermatic cord. Neoplastic markers showed high levels of CEA (carcinoembryonic antigen) and bHCG (beta Human Chorionic Gonadotropin). The patient underwent right surgical scrotal exploration with orchifunicolectomy. Pathologic examination revealed pathologic tissue showing rare glandular structures. Immunohistochemistry profile was compatible with malign epithelial neoplasm with glandular differentiation. Total body CT-scan revealed pathologic tissue in pancreas between head and body and a suspect pathologic lesion in liver and 18-FDG PET-scan confirmed the pancreatic neoplastic mass and a suspect secondary hepatic lesion. Biopsy of pancreatic pathologic area was positive for ductal pancreatic adenocarcinoma. The patient was sent to oncologic evaluation and started chemotherapy.

Conclusions: Malignancies of epididymis and spermatic cord are rare entities and, in literature, very low number of cases of metastasis from pancreatic carcinoma to epididymis and spermatic cord are described. Early differential diagnosis is fundamental mostly in those patients with age range unusual for testis cancers.
\end{abstract}

KEY WORDS: Spermatic cord cancer; Metastasis; Pancreatic cancer; Adenocarcinoma; Epididymis cancer; Scrotal tumour.

Submitted 6 January 2018; 24 February 2018

\section{INTRODUCTION}

Metastatic epididymal and spermatic cord adenocarcinoma from epithelial tumors are a rare condition. The most frequent primary cancers with metastasis to epididymis and spermatic cord are prostate, lung, kidney, gastrointestinal tumors and breast cancers (1-2). In literature, there are few reports regarding epididymis and spermatic cord metastasis in gastric cancers (3-5), colorectal cancers (6-8), prostate cancer (9) and pancreatic adenocarcinoma (2). In particular, very low number of cases reported metastasis from pancreatic cancer to epididymis and spermatic cord. We report a case of 70-years old man with history of left orchiectomy for undescended testicle in young age, who presented to our department with a palpable nodule in right scrotum. The patient underwent right radical orchifunicolectomy finding adenocarcinoma of epididymis and spermatic cord probably secondary to gastrointestinal cancer; a total body CT-scan showed a suspect pancreatic lesion that a biopsy confirmed to be a ductal pancreatic adenocarcinoma.

\section{Case report}

A 70-years old man presented with right scrotal palpable nodule without pain or other symptoms, with history of left orchiectomy in young age for undescended testis. Scrotal ultrasound revealed an inhomogeneous hypoechoic nodule of epididymis and/or spermatic cord with small calcifications. Neoplastic markers showed carcinoembryonic antigen (CEA) and beta Human chorionic gonadotropin (bHCG) respectively $9,7 \mathrm{ng} / \mathrm{ml}$ (reference $\leq$ $5.5 \mathrm{ng} / \mathrm{ml}$ ) and $6.7 \mathrm{mUI} / \mathrm{ml}$ (reference $\leq 2.5 \mathrm{mUI} / \mathrm{ml}$ ). The patient underwent right surgical scrotal exploration with orchifunicolectomy.

Pathologic examination revealed a neoplastic mass of around 2 centimetres at epididymis and spermatic cord. Microscopically, pathologic tissue showed rare glandular structures, with slit-like and papillary pattern and presence of mitosis with associated desmoplastic reaction. Immunohistochemistry profile was positive for cytokeratin AEl AE3, polyclonal CEA, cytokeratin 7, BerEp4 (Ep-CAM/ Epithelial Specific Antigen) and negative for alpha-fetoprotein, OCT3/4 (octamer-binding transcription factor), D240, Placental Alkaline Phosphatase (PLAP), CD30. This histopathologic pattern was compatible with malign epithelial neoplasm with glandular differentiation. Based on rarity of primary epididymis and spermatic cord 


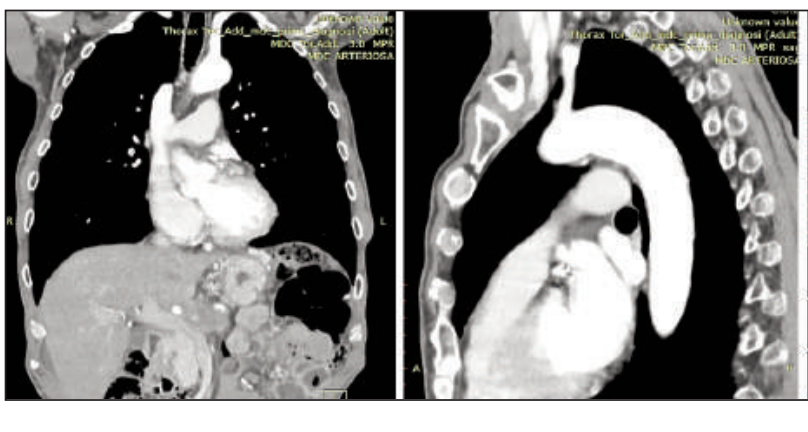

Figure 2.

18 FDG PET-scan showed a pancreatic metabolic lesion compatible with neoplastic mass.

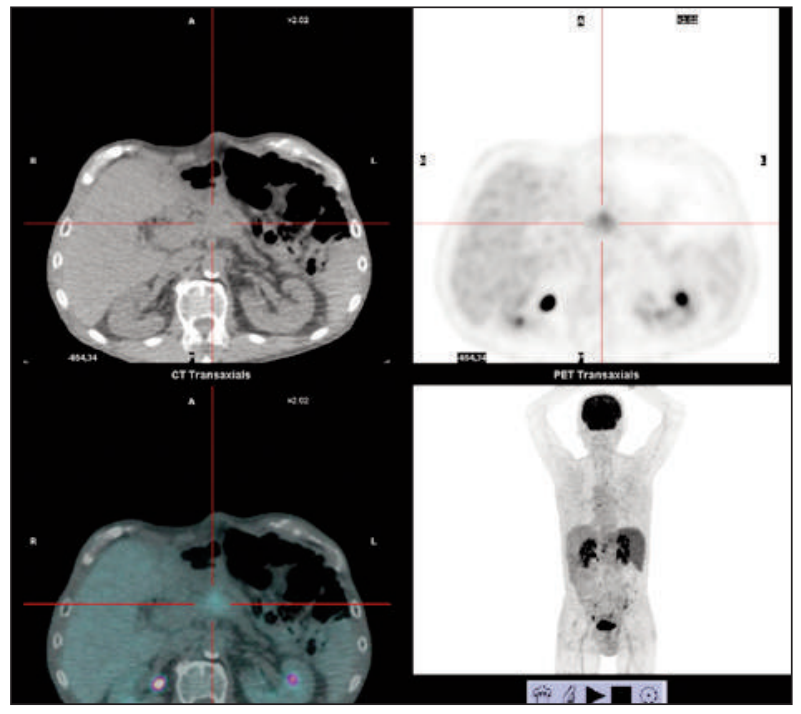

tumors, the Pathologist suggested a differential diagnosis with other epithelial cancers. We performed a total body CT-scan that revealed pathologic tissue in pancreas between head and body and a suspect pathologic lesion in the left hepatic lobe of 26 millimetres and another suspect metastatic hepatic lesion in VII segment of 12 millimetres (Figure 1). 18-FDG PET-scan confirmed the pancreatic neoplastic mass and a suspect secondary hepatic lesion (Figure 2). Patient performed endoscopic ultrasound and biopsy of pancreatic pathologic area that was positive for ductal pancreatic adenocarcinoma. The patient was sent to oncologic evaluation and started chemotherapy with Gemcitabine and Abraxane i.v according the scheme day $1,8,15$ of a 28 days cycle.

\section{Conclusion}

Generally, malignancies of epididymis and spermatic cord are rare entities, with few cases of both primary cancers and secondary ones. In literature, very low number of cases of metastasis from pancreatic carcinoma to epididymis and spermatic cord are described and usually they are associated with a poor prognosis. Early differential diagnosis is fundamental mostly in those patients with age range unusual for testis cancers. In these cases, at the time of diagnosis, it would be correct to suspect and exclude an "extra-scrotal" origin of the disease.

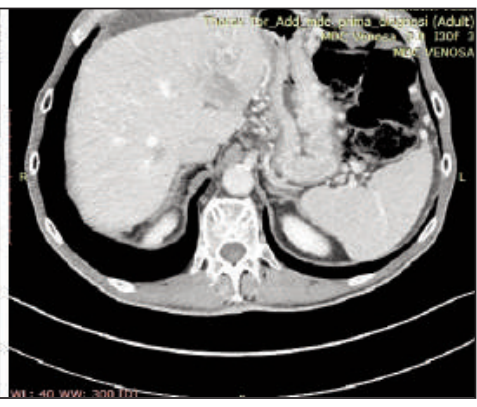

Figure 1.

Contrast CT-scan showed a pancreatic neoplastic lesion between the pancreatic head and body and suspect hepatic secondary lesions.

\section{References}

1. Algad F, Santaularia JM, Villavicencio H. Metastatic tumour of the epididymis and spermatic cord. Eur Urol. 1983; 9:56-9.

2. Kanno K, Ohwada S, Nakamura S, et al. Epididymis metastasis from colon carcinoma: A case report and a review of the Japanese literature. Jpn J Clin Oncol. 1994; 24:340-4.

3. Irisawa C, Yamaguchi O, Shiraiwa Y, et al. A case of metastatic tumor of the spermatic cord from gastric carcinoma. Hinyokika Kiyo. 1989; 35:1807-1809.

4. Pozzobon D, Caldato C, Pavanello M, Di Falco G. Metastatic gastric neoplasm in the spermatic cord: report of a case. Chirltal. 2001; 53:729-32.

5. Schaefer I. M, Sauer U, Liwocha M, et al. Occult gastric signet ring cell carcinoma presenting as spermatic cord and testicular metastases: "Krukenberg tumor" in a male patient. Pathol Res Pract. 2010; 206:519-521.

6. Shida Y, Miyata Y, Igawa T, et al. A case of metastatic tumor of spermatic cord from ascending colon carcinoma. Hinyokika Kiyo. 2006; 52:733-5.

7. Polychronidis A, Tsolos C, Sivridis E, et al. Spermatic cord metastasis as an initial manifestation of sigmoid colon carcinoma: Report of a case. Surg Today. 2002; 32:376-7.

8 Melone F, Olmastroni M, Petacchi D, et al. Metastatic tumor of the spermatic cord from a primary silent colorectal adenocarcinoma. Minerva Urol Nefrol. 1997; 49:57-61.

9. Bawa AS, Singh R, Bansal VK, Punia RS. Spermatic cord metastasis from prostatic cancer. J Postgrad Med. 2003; 49:97-98.

\section{Correspondence}

Carmelo Agostino Di Franco, MD (Corresponding Author) carmelo_difranco@tiscali.it

Bruno Rovereto, $M D$

Daniele Porru, MD

Valeria Zoccarato, MD

Cesare Regina, MD

Tiziano Cebrelli, MD

Nicolò Fiorello, MD

Carlo Marchetti, MD

Andrea Ringressi, MD

Davide Barletta, MD

Giovanni Giliberto, MD

Bruno Rovereto, $M D$

Department of Urology, University Hospital IRCCS Policlinico S.Matteo, Pavia, Italy

Alessandra Viglio, MD

Department of Pathology, University Hospital IRCCS Policlinico S.Matteo, Pavia, Italy

Lavinia Galvagno, Prof.

Language and Translation Services, Enna, Italy 